\title{
EQUALITY OF ESSENTIAL SPECTRA OF QUASISIMILAR OPERATORS WITH PROPERTY $(\delta)$
}

\author{
by T. L. MILLER and V. G. MILLER
}

(Received 10 May, 1994; revised 4 November, 1994)

\begin{abstract}
A Banach space operator has property $(\delta)$ if and only if it is the quotient of a decomposable operator, equivalently, if and only if its adjoint has Bishop's property $(\beta)$. Within this class of operators, it is shown that quasisimilarity preserves essential spectra.
\end{abstract}

0. Introduction. A continuous linear operator $T$ on a complex Banach space $X$ is said to be a Fredholm operator provided that the kernel of $T$ is finite dimensional and the range of $T$ is finitely complemented in $X$; that is, there is a finite dimensional subspace $F$ of $X$ so that $X$ is the algebraic direct sum of $F$ and $\operatorname{ran}(T)$. This second condition is equivalent to the requirements that $\operatorname{ran}(T)$ be closed and that the quotient $X / \operatorname{ran}(T)$ be finite dimensional, [8, Proposition 36.3]. By the essential spectrum of $T$ we mean the set of $\lambda \in \mathbb{C}$ such that $T-\lambda$ is not a Fredholm operator.

Two continuous linear operators $T \in \mathscr{L}(X)$ and $Y \in \mathscr{L}(Y)$ on complex Banach spaces $X$ and $Y$ are called quasisimilar if there exist $A \in \mathscr{L}(X, Y)$ and $B \in \mathscr{L}(Y, X)$, each injective, and with dense range, so that $A T=S A$ and $T B=B S$. The invariance of various spectra under this equivalence relation for certain classes of operators has received considerable attention. We mention in particular that quasisimilar hyponormal operators were shown by S. Clary [3] to have equal spectra, and that L. R. Williams [18] showed that quasisimilar quasinormal operators have equal essential spectra. L. Yang [19] generalized Williams's work to the class of subnormal operators.

In 1984, M. Putinar [14] constructed a functional model for hyponormal operators that showed them to be subscalar and hence to possess a certain property introduced by E. Bishop [2]: a Banach space operator $T \in \mathscr{L}(X)$ is said to have property $(\beta)$ provided that whenever a sequence of analytic functions $f_{n}: U \rightarrow X$ is such that $(T-\lambda) f_{n}(\lambda) \rightarrow 0$ uniformly on the compact subsets of an open subset $U$ of $\mathbb{C}$, it follows that $f_{n}(\lambda) \rightarrow 0$ uniformly on the compact subsets of $U$. Putinar must be given credit as one of the first to recognize the importance of $(\beta)$ in localizing the analytic functional calculus of an operator and the corresponding decomposition of its spectrum.

Putinar's work on hyponormal operators led Yang [20] to show equality of essential spectra of quasisimilar hyponormal, and more generally, restrictions of decomposable operators on Hilbert spaces. The class of decomposable operators, defined below, includes generalized scalar operators and normal operators and shares many of the spectral decomposition properties of normal operators, in particular those on which Yang's proof [19] of the subnormal case were based. Using his sheaf-theoretic model for Banach space operators with property $(\beta)$ [13] Putinar showed that quasisimilar tuples of operators with $(\beta)$ have equal spectra and essential spectra [15].

The functional model for hyponormal operators led E. Albrecht and J. Eschmeier [1] to construct a complete duality theory for operators with Bishop's property $(\beta)$. Recall that an operator $T \in \mathscr{L}(X)$ is decomposable in the sense of $\mathrm{C}$. Foiaş provided that for

Glasgow Math. J. 38 (1996) 21-28. 
every open cover $\left\{U_{1}, U_{2}\right\}$ of the complex plane $\mathbb{C}$, there exist closed $T$-invariant subspaces $X_{1}$ and $X_{2}$ of $X$ so that $X=X_{1}+X_{2}$ and $\sigma\left(\left.T\right|_{X_{i}}\right) \subset U_{i}$ for $i=1,2$. See [4] and [17]. Albrecht and Eschmeier show that $(\beta)$ characterizes restrictions of decomposable operators to closed invariant subspaces, and that quotients of decomposable operators are determined by the decomposition property $(\delta): T \in \mathscr{L}(X)$ is said to have property $(\delta)$ provided that for each open cover $\left\{U_{1}, U_{2}\right\}$ of the complex plane $\mathbb{C}$, there exist $T$-invariant linear manifolds $X_{1}$ and $X_{2}$ of $X$ so that $X=X_{1}+X_{2}$ and such that for each $x \in X_{i}$ there is an analytic function $f: \mathbb{C} \backslash \bar{U}_{i} \rightarrow X$ with $(T-\lambda) f(\lambda)=x$ for all $\lambda \in \mathbb{C} \backslash \bar{U}_{i}$. Albrecht and Eschmeier further show that the properties $(\beta)$ and $(\delta)$ are completely dual: an operator has one if and only if its adjoint has the other.

It is a consequence of [12, Proposition 2.4] that quasisimilar operators with property ( $\delta$ ) have equal spectra. Since an operator $T$ is Fredholm if and only if $T^{*}$ is also, it is natural to conjecture that quasisimilar Banach space operators with property $(\delta)$ have equal essential spectra. Indeed, in the case of reflexive spaces, the result follows immediately from Putinar's result; see [15]. The purpose of this note is to establish this equality in the setting of arbitrary Banach spaces. In fact, we show somewhat more.

TheOREM. Suppose that $T \in \mathscr{L}(X)$ and $S \in \mathscr{L}(Y)$ each have the decomposition property ( $\delta$ ). If $A \in \mathscr{L}(X, Y)$ and $B \in \mathscr{L}(Y, X)$ are each injective, if $A T=S A$ and $T B=B S$, then $\sigma_{\mathrm{e}}(T)=\sigma_{\mathrm{e}}(S)$.

In the first section we present the elements of local spectral theory on which the proof of the theorem in Section 2 is based.

1. Preliminaries. If $T$ is a continuous linear operator on a Banach space $X$, we denote the spectrum of $T \in \mathscr{L}(X)$ by $\sigma(T)$. By the surjectivity spectrum of $T$, we mean $\sigma_{s u}(T)=\{\lambda: T-\lambda$ is not surjective $\}$; let $\sigma_{p}(T)$ and $\sigma_{e}(T)$ denote respectively the point spectrum and essential spectrum of $T$. The resolvent set of $T$ is $\rho(T)=\mathbb{C} \backslash \sigma(T)$; let $\rho_{e}(T)=\mathbb{C} \backslash \sigma_{e}(T)$ and $\rho_{s u}(T)=\mathbb{C} \backslash \sigma_{s u}(T)$. If $E$ is a locally convex topological vector space, we denote the dual of $E$ by $E^{*}$ and call the topology on $E^{*}$ determined by $E$ the weak-* topology; that is, $\varphi_{\alpha} \stackrel{\text { weak-* }}{\longrightarrow} \varphi$ in $E^{*}$ if $\varphi_{\alpha}(x) \rightarrow \varphi(x)$ for each $x \in E$. If $T$ is a continuous linear operator on $E$, let $\operatorname{ker}(T)$ and $\operatorname{ran}(T)$ denote respectively the kernel and range of $T$. For $M$ a closed subspace of $E$, let $\left.T\right|_{M}$ be the restriction of $T$ to $M$. If $T \in \mathscr{L}(E)$ and if $M$ is a $T$-invariant subspace of $E$, we consider $\left.T\right|_{M}: M \rightarrow M$.

For a Banach space $X$ and an open subset $V$ of the plane $\mathbb{C}$, let $\mathcal{O}(V, X)$ denote the space of analytic $X$-valued functions on $V$. Observe that $O(V, X)$ is a Fréchet space when endowed with the topology determined by uniform convergence on the compact subsets of $V$.

If $U$ is open in the extended plane $\mathbb{C}_{\infty}$ with $\infty \in U$, let $P(U, X)$ denote the set of analytic $X$-valued functions $f$ on $U$ such that $f(\infty)=0$. This is also a Fréchet space when endowed with the topology determined by uniform convergence on the compact subsets of $U$. If $F \subset \mathbb{C}_{\infty}$ is closed with $\infty \in F$, let $P(F, X)$ be the inductive limit of the collection $\{P(U, X): U$ an open neighborhood of $F\}$. Here, we identify functions that agree in a neighborhood of $F$. The space $P(F, X)$ is a complete (LF)-space [7, Proposition 15], and for $V$ an open subset of $\mathbb{C}, O(V, X)^{*}$ is isomorphic to $P\left(\mathbb{C}_{\infty} \backslash V, X^{*}\right)$, and $P\left(\mathbb{C}_{x} \backslash V, X\right)^{*}$ is isomorphic to $O\left(V, X^{*}\right)\left[7\right.$, Proposition 13]. (Here $P\left(\mathbb{C}_{x} \backslash V, X\right)^{*}$ and $O(V, X)^{*}$ are equipped with their strong dual topologies.) 
If $U$ is open in $\mathbb{C}_{x}$ about $\propto, 0 \notin U$, let $V=\{1 / z: z \in U\}, K=\mathbb{C} \backslash U$, and $F=\mathbb{C}_{x} \backslash V$. The mapping $f(z) \mapsto f(1 / z)$ is an isomorphism between $P(U, X)$ and $\{f \in O(V, X): f(0)=0\}$. Thus, from the duality result above, $P(U, X)^{*}$ is isomorphic to $O\left(K, X^{*}\right)$, the inductive limit of $\left\{O\left(W, X^{*}\right): K \subset W\right\}$, where again functions agreeing on a neighborhood of $K$ are identified. The space $\mathcal{O}\left(K, X^{*}\right)$ is also a complete (LF)-space.

Let $F$ be closed in $\mathbb{C}_{x}$ with $\propto \in F$ and $U$ an open neighborhood of $F$. If $T \in \mathscr{L}(X)$, define $T^{U}: P(U, X) \rightarrow P(U, X)$ by $\left(T^{U} f\right)(\lambda)=(T-\lambda) f(\lambda)+\lim _{z \rightarrow \infty} z f(z), \lambda \in U$, and let $T^{F}$ be the corresponding operator on $P(F, X)$. Similarly, for $K$ compact and $V$ open with $K \subset V \subset \mathbb{C}$, define $T_{V}$ on $\mathcal{O}(V, X)$ by $\left(T_{V} f\right)(\lambda)=(T-\lambda) f(\lambda)$, and $T_{K}$ on $\mathcal{O}(K, X)$ by $\left.T_{K}\right|_{\alpha v, x)}=T_{V}$. Each of these mappings is continuous; also by $[5,1.3 .4]$, for $U$ and $F$ as above, we have

$$
\left(T^{U}\right)^{*}=T_{\mathbb{C} U}^{*}, \text { and }\left(T^{F}\right)^{*}=T_{\mathbb{C} V}^{*}
$$

Similarly, if $K \subset V$ are as above, then

$$
\left(T_{V}\right)^{*}=\left(T^{*}\right)^{\mathbb{C}_{x} V}, \text { and }\left(T_{K}\right)^{*}=\left(T^{*}\right)^{\mathbb{C}_{x} K} \text {. }
$$

If $V$ is open in the plane and $T_{V}$ has closed range, we denote the Fréchet space $O(V, X) / \operatorname{ran}\left(T_{V}\right)$ by $\mathscr{F}_{T}(V)$. For $K$ a compact subset of the plane such that $T_{K}$ has closed range, we similarly let $\mathscr{F}_{T}(K)=\mathscr{O}(K, X) / \operatorname{ran}\left(T_{K}\right)$, the inductive limit of the spaces $\mathscr{F}_{T}(W)$, where $W$ runs through the open neighborhoods of $K$.

An operator $T$ is said to have the single-valued extension property (SVEP) provided that $T_{V}$ is injective for each open subset $V$ of the plane. Bishop's property $(\beta)$ may be restated as the condition that for each open subset $V$ of $\mathbb{C}$ the operator $T_{V}$ is injective and has closed range. If $T$ has property $(\beta)$ and $V$ is open in the plane, $\mathscr{F}_{T}(V)$ is the presheaf corresponding to Putinar's sheaf model for an operator with property $(\beta)$; see $[13]$. Since we are concerned only with a single operator, we can avoid using this theory explicitly.

For $T \in \mathscr{L}(X)$ and $H$ closed in $\mathbb{C}$, we define a $T$-invariant, but generally not closed, subspace of $X, \ddot{x}_{T}(H)=\left\{x \in X: x \in \operatorname{ran}\left(T_{\mathbb{C} H}\right)\right\}$. Clearly, the operator $T$ has property $(\delta)$ provided that whenever $U$ and $V$ are open with $U \cup V=\mathbb{C}$, the space $X$ can be written as $X=\mathfrak{X}_{T}(\bar{U})+\mathfrak{X}_{T}(\bar{V})$. Equivalently, by $[5$, I.3.4], $T \in \mathscr{L}(X)$ has property $(\delta)$ if and only if for each closed $F$ in $\mathbb{C}_{x}$ with $x \in F$, the mapping $T^{F}$ is surjective. If $T$ has property $(\delta)$, it follows from $[10,34.8 .(4)]$ that $T^{F}$ is an open mapping and therefore by [10,32.3.(1)], that its adjoint $T_{U}^{*}$ has weak-* closed range. Thus $\left(\operatorname{ker}\left(T^{F}\right)\right)^{*}=\mathscr{F}_{T^{*}}(U)$.

2. Proof of the Theorem. For $\lambda \in \mathbb{C}$ and $\epsilon>0$, let $V(\lambda, \epsilon)$ denote the open ball of radius $\epsilon$ centered at $\lambda$.

Lemma 1. Suppose that $T \in \mathscr{L}(X)$ is surjective and Fredholm. Then there exists an $\epsilon>0$ such that $T_{V(0, \epsilon)}^{*}$ has closed range and $\operatorname{dim} \operatorname{ker}(T-\lambda)=\operatorname{dim} \operatorname{ker}(T)$ for all $\lambda \in V:=V(0, \epsilon)$. Let $n=\operatorname{dim} \operatorname{ker}(T)$, and assume that $n>0$.

(1) There exist $\left\{g_{j}\right\}_{j=1}^{n} \subset \mathcal{O}(V, X)$ and $\left\{f_{j}\right\}_{j=1}^{n} \subset \mathcal{O}\left(V, X^{*}\right)$ satisfying the following conditions for each $\lambda \in V$.

(i) The set $\left\{g_{j}(\lambda)\right\}_{1}^{n}$ is a basis for $\operatorname{Ker}(T-\lambda)$.

(ii) $\left[f_{j}(\lambda)\right]_{\lambda}:=f_{j}(\lambda)+\operatorname{ran}\left(T^{*}-\lambda\right), 1 \leq j \leq n$, is a basis for $X^{*} / \operatorname{ran}\left(T^{*}-\lambda\right)$.

(iii) $f_{j}(\lambda)\left(g_{i}(\lambda)\right)=1$ if $i=j$ and 0 otherwise. 
Moreover, if $W$ is an open subset of $V$, and $f \in \mathcal{O}\left(W, X^{*}\right)$, then there exist unique analytic functions $\varphi_{W_{j}}: \mathbb{C}, 1 \leq j \leq n$, and some $g \in \mathcal{O}\left(W, X^{*}\right)$ such that

$$
f=\sum_{j=1}^{n} \varphi_{W j} f_{j}+T_{w}^{*} g
$$

(2) If $C \in \mathscr{L}(X)$ is injective and such that $T C=C T$, then for each open set $W \subset V$, the induced mapping

$$
\mathscr{F}_{T^{*}}(W) \stackrel{\left[C^{*}\right]}{\longrightarrow} \mathscr{F}_{T^{*}}(W), \quad[f] \mapsto\left[C^{*} \circ f\right],
$$

is a continuous bijection. It follows that for $K$ a compact subset of $V$ such that $\operatorname{ran}\left(T_{K}^{*}\right)$ is closed, $\left[C^{*}\right]$ is a continuous bijection on $\mathscr{F}_{T^{*}}(K)$ as well.

Proof. The first part of the following lemma is well-known; see for example Proposition 3 and its Corollary in [16]. To see (2), let $W$ be an open subset of $V$. The mapping $C^{*} \circ$ is clearly continuous on $\mathcal{O}\left(W, X^{*}\right)$, and therefore the quotient mapping $\left[C^{*}\right]$ is continuous on $\mathscr{F}_{T^{*}}(W)$. If $f_{1}, \ldots, f_{n} \in \mathcal{O}\left(V, X^{*}\right)$ are as in (1), then for each $i$ and $j$, $1 \leq i, j \leq n$, there exist unique analytic functions $\varphi_{i j}: V \rightarrow \mathbb{C}$ such that for each $j$, $\left[C^{*}\right]\left[f_{j}\right]=\sum_{i=1}^{n} \varphi_{i j}\left[f_{i}\right]$ in $\mathscr{F}_{T^{*}}(V)$. Since $C$ is injective on $X$ and $\operatorname{ker}(T-\lambda)$ is finite dimensional, $C: \operatorname{ker}(T-\lambda) \rightarrow \operatorname{ker}(T-\lambda)$ is a bijection for each $\lambda \in V$. Consequently, the dual mapping, $\left[C^{*}\right]_{\lambda}: X^{*} / \operatorname{ran}\left(T^{*}-\lambda\right) \rightarrow X^{*} / \operatorname{ran}\left(T^{*}-\lambda\right)$ is a bijection. It follows that $\operatorname{det}\left(\varphi_{i j}(\lambda)\right) \neq 0$, and therefore that $\left[C^{*}\right]$ is a bijection on $\mathscr{F}_{T^{*}}(W)$.

Now, if $K$ is a compact subset of $V$, then for each open neighborhood $W$ of $K, C^{*} \circ$ is continuous on $O\left(W, X^{*}\right)$, and therefore $C^{*} \circ$ is continuous with respect to the inductive limit topology on $\mathcal{O}\left(K, X^{*}\right)$. Under the assumption that $\operatorname{ran}\left(T_{K}^{*}\right)$ is closed, the quotient mapping is continuous as well. Let $f \in \mathcal{O}\left(K, X^{*}\right)$, and choose $W$ open, with $K \subset W \subset V$, such that $f \in \mathcal{O}\left(W, X^{*}\right)$. Since $\left[C^{*}\right]$ is a bijection on $\mathscr{F}_{T^{*}}(W)$, there is a unique $g$ in $\mathcal{O}\left(W, X^{*}\right)$ so that $f=C^{*} g+T_{W}^{*} h$ for some $h \in O\left(W, X^{*}\right)$; in particular, $f+\operatorname{ran}\left(T_{K}^{*}\right)=$ $C^{*} g+\operatorname{ran}\left(T_{K}^{*}\right)$ and thus $\left[C^{*}\right]$ is surjective. Since $\left[C^{*} f\right] \in \operatorname{ran}\left(T_{K}^{*}\right)$ implies that $\left[C^{*} f\right] \in$ $\operatorname{ran}\left(T_{W}^{*}\right)$ for some $W$ open about $K$, it follows that $\left[C^{*}\right]$ is a bijection on $\mathscr{F}_{T^{*}}(K)$.

Lemma 2. Let $T$ and $V=V(0, \epsilon)$ be as in Lemma 1 , and let $F=\mathbb{C}_{x} \backslash V$. Then $T^{F}$ is surjective on $P(F, X)$, and consequently, its dual $T_{V}^{*}$ has weak-* closed range in $O\left(V, X^{*}\right)$. Moreover, if $C \in \mathscr{L}(X)$ is injective and commutes with $T$, then the mapping $f \mapsto C \circ f$ is a continuous bijection on $\operatorname{ker}\left(T^{F}\right)$.

Proof. Denote the mapping $f \mapsto C \circ f$ by $C$. For each open set $U \supset F$, it's clear that $C$ is continuous and injective on $P(U, X)$, and therefore $C: P(F, X) \rightarrow P(F, X)$ is injective and continuous. Since $T$ is Fredholm and surjective, $T$ is right-invertible; say $L \in \mathscr{L}(X)$ is such that $T L=I$. By shrinking $\epsilon$ if necessary, we may assume that $\epsilon<\|L\|^{-1}$.

For $0<\delta<\epsilon$, let $U_{\delta}=\mathbb{C} \backslash V(0, \delta)$. Since

$$
\begin{gathered}
P(F, X)=\bigcup_{\delta<\epsilon} P\left(U_{\delta}, X\right), \\
\operatorname{ker}\left(T^{F}\right)=\bigcup_{\delta<\epsilon}\left(P\left(U_{\delta}, X\right) \cap \operatorname{ker}\left(T^{F}\right)\right)=\bigcup_{\delta<\epsilon} \operatorname{ker}\left(T^{U_{\delta}}\right),
\end{gathered}
$$

we need only show that $T^{U_{\delta}}$ and $C$ are surjective for each $\delta<\epsilon$. Fix such a $\delta$; let $U=U_{\delta}$ 
and $K=\mathbb{C} \backslash U_{\delta}$. A function $f \in P(U, X)$ has a Laurent series $f(z)=\sum_{1}^{\infty} a_{n} / z^{n}$ where $\left(a_{n}\right)_{n} \subset X$ is such that $\varlimsup_{n \rightarrow \infty}\left\|a_{n}\right\|^{1 / n} \leq \delta$. For each $n$, define $b_{n}=\sum_{k=n}^{\infty} L^{k-n+1} a_{k}$ and set $g(z)=\sum_{1}^{\infty} b_{n} / z^{n}$. It follows routinely that $\varlimsup_{n \rightarrow \infty}\left\|b_{n}\right\|^{1 / n} \leq \delta$, and thus $g \in P(U, X)$ with $T^{U} g=f$. Because $T^{F}$ is surjective on the (LF)-space $P(F, X)$, it is an open mapping $[10,34.8$.(4)] and therefore by $[10,32.3 .(1)]$ its dual has weak-* closed range.

Since $T^{U}$ is similarly an open mapping, $T_{K}^{*}=\left(T^{U}\right)^{*}$ also has weak-* closed range in $O\left(K, X^{*}\right)$, and so $\operatorname{ker}\left(T^{U}\right)^{*}=\mathscr{F}_{T^{*}}(K)$. By Lemma $1,\left[C^{*}\right]: \mathscr{F}_{T^{*}}(K) \rightarrow \mathscr{F}_{T^{*}}(K)$, the dual of $C: \operatorname{ker}\left(T^{U}\right) \rightarrow \operatorname{ker}\left(T^{U}\right)$, is a bijection. It follows from the closed range theorem for Fréchet spaces, $\left[9,9.6 .3\right.$, p. 185], that $C\left(\operatorname{ker}\left(T^{U}\right)\right)$ is closed, and therefore a bijection as well.

For $V$ open in the plane, let $\mathfrak{X}_{T}(V)=\cup\left\{\mathfrak{X}_{T}(H): H\right.$ a closed subset of $\left.V\right\}$. If $F$ is closed in $\mathbb{C}_{\alpha}$ with $\infty \in F$ and if $V=\mathbb{C}_{\alpha} / F$, it is straightforward to check that $\tau: P(F, X) \rightarrow$ $X, \tau(f)=-\lim _{z \rightarrow \infty} z f(z)$ is a bijection between $\operatorname{ker}\left(T^{F}\right)$ and $\mathfrak{X}_{T}(V)$; see [1,p. 12]. Since $C \tau=\tau C$, we immediately obtain the following result.

COROLlary. Assume that $T \in \mathscr{L}(X)$ is a surjective Fredholm operator. If $C$ is injective and commutes with $T$, then for all sufficiently small $\epsilon>0$ we have

$$
C\left(\mathfrak{X}_{T}(V(0, \epsilon))\right)=\mathfrak{X}_{T}(V(0, \epsilon)) \text {. }
$$

Remark. The spectral subspaces $\mathfrak{X}_{T}(V(0, \epsilon))=\left\{x \in X: \varlimsup_{n \rightarrow \infty}\left\|T^{n} x\right\|^{1 / n}<\epsilon\right\}$ ([12, Proposition 2.1]) can be large. Consider the Hardy space $H^{2}=\left\{f \in L^{2}[0,2 \pi]: \hat{f}(n)=0\right.$ if $\left.n<0\right\}$, where for each integer $n$, the $n$th Fourier coefficient of $f \in L^{2}[0,2 \pi]$ is given by $\hat{f}(n)=$ $\frac{1}{2 \pi} \int_{0}^{2 \pi} f\left(e^{i \theta}\right) e^{-i n \theta} d \theta$. Let $T^{*}=M_{z}$, multiplication by the identity function $z \mapsto z$; so that $T=M_{z}^{*}=P_{H^{2}} M_{\bar{z}}$. Then $T^{*}$ is an isometry with range $\left\{f \in H^{2}: f^{\wedge}(0)=0\right\}$, and with $\sigma_{e}\left(T^{*}\right)=\{\lambda:|\lambda|=1\}$. If $0<\epsilon \leq 1$, then the spectral subspace $\mathfrak{S}_{T}^{2}(V(0, \epsilon))$ contains $\left\{z^{n}\right\}_{n=0}^{\infty}$ and is therefore dense in $H^{2}$.

An operator $C \in \mathscr{L}\left(H^{2}\right)$ commutes with $T$ if and only if $C=M_{\varphi}^{*}$, where $M_{\varphi} f=\varphi f$ and $\varphi$ is a bounded analytic function on $D=V(0,1)$. By Beurling's Theorem, such a $C$ is injective if and only if $\varphi$ is outer; i.e.,

$$
\varphi(z)=\exp \left(\frac{1}{2 \pi} \int_{0}^{2 \pi} \frac{e^{i \theta}+z}{e^{i \theta}-z} \log \left|\varphi\left(e^{i \theta}\right)\right| d \theta\right) .
$$

In particular, if $\varphi$ is outer, $M_{\varphi}$ is injective on $H^{2}$; equivalently, $C=M_{\varphi}^{*}$ has dense range in $H^{2}$.

In this context, the corollary asserts that if $\varphi$ is an outer function in $H^{\infty}(D)$ and if $f \in H^{2}$ is such that $\varlimsup_{n \rightarrow \infty}\left\|\left(M_{z}^{*}\right)^{n} f\right\|<1$, then $f=M_{\varphi g}^{*} g$ for some $g \in H^{2}$.

Lemma 3. Suppose that $T$ and $V=V(0, \epsilon)$ are as in Lemma 1 ; specifically, $T \in \mathscr{L}(X)$ is Fredholm and surjective. Assume further that $S \in \mathscr{L}(Y)$ has property $(\delta)$, and is such that there exist injective operators $A \in \mathscr{L}(X, Y)$ and $B \in \mathscr{L}(Y, X)$ satisfying $A T=S A$ and 
$B S=T B$. Then the mappings $\left[B^{*}\right]: \mathscr{F}_{T^{*}}(V) \rightarrow \mathscr{F}_{S^{*}}(V)$ and $\left[A^{*}\right]: \mathscr{F}_{S^{*}}(V) \rightarrow \mathscr{F}_{T^{*}}(V)$ are bijections, and $S^{*}$ is injective.

Proof. Let $F=\mathbb{C}_{\infty} \backslash V$. Because $A$ and $B$ are injective and intertwine $T$ and $S, B A$ satisfies the hypotheses of Lemma 2, and therefore $B A: \operatorname{ker}\left(T^{F}\right) \rightarrow \operatorname{ker}\left(T^{F}\right)$ is a continuous bijection. If $f \in \operatorname{ker}\left(S^{F}\right)$, then $B f \in \operatorname{ker}\left(T^{F}\right)$, and thus there is a unique $g \in \operatorname{ker}\left(T^{F}\right)$ such that $B f=B A g$. Since $B$ is injective on $\operatorname{ker}\left(S^{F}\right)$, it follows that $A: \operatorname{ker}\left(T^{F}\right) \rightarrow \operatorname{ker}\left(T^{F}\right)$ is surjective and therefore by $[10,32.7]$ the dual map, $\left[A^{*}\right]: \mathscr{F}_{S^{*}}(V) \rightarrow \mathscr{F}_{T^{*}}(V)$ is injective. Since $\left[A^{*}\right]\left[B^{*}\right]=\left[A^{*} B^{*}\right]$ is a bijection, it follows that both $\left[A^{*}\right]$ and $\left[B^{*}\right]$ are bijective as well.

If $y^{*} \in \operatorname{ker}\left(S^{*}\right)$, then $A^{*} y^{*} \in \operatorname{ker}\left(T^{*}\right)=\{0\}$, and therefore $\left[A^{*}\right]\left[y^{*}\right]=0$. Since $\left[A^{*}\right]$ is a bijection, we must have $\left[y^{*}\right]=0$; say $g \in \mathcal{O}\left(V, Y^{*}\right)$ is such that $\left(S^{*}-\lambda\right) g(\lambda)=y^{*}$ for each $\lambda \in V$. However $y^{*}=\left(S^{*}-\lambda\right)\left(-\frac{1}{\lambda} y^{*}\right)$ for every $\lambda \neq 0$. Because $S^{*}$ has property $(\beta)$, in particular the single valued extension property, it follows that $g(\lambda)=$ $-\frac{1}{\lambda} y^{*}$ on $V \backslash\{0\}$. Thus $g \in O\left(V, Y^{*}\right)$ implies that $y^{*}=0$.

If $T$ is a Fredholm operator on $X$, recall that $T^{n}$ is also Fredholm for each positive integer $n$; see [8, Proposition 25.3].

LEMma 4. If $T \in \mathscr{L}(X)$ is Fredholm and has the single valued extension property, then there is a neighborhood $U$ of 0 and a positive integer $n$ such that $\left.(T-\lambda)\right|_{\operatorname{ran}\left(T^{n}\right)}$ is injective for each $\lambda \in U$.

Proof. Under the assumptions on $T$, [6, Theorem 9] implies that no neighborhood of 0 is contained in $\sigma_{p}(T)$. It now follows from [8, Proposition 51.2] that there is a neighborhood $U$ of 0 such that $\sigma_{p}(T) \cap U \subset\{0\}$. If $Z$ is the closed $T$-invariant subspace $\cap_{n \geq 1} T^{n} X$, then $\left.T\right|_{z}$ is a bijection by [8, Proposition 38.7]. Because $\operatorname{ker}(T)$ is finite dimensional, and $\operatorname{ran}\left(T^{n}\right) \cap \operatorname{ker}(T)$ is decreasing to $\{0\}=Z \cap \operatorname{ker}(T)$, there is an $n$ such that $\operatorname{ran}\left(T^{n}\right) \cap \operatorname{ker}(T)=\{0\}$. It follows that $\left.(T-\lambda)\right|_{\operatorname{ran}\left(T^{n}\right)}$ is injective for each $\lambda \in U$.

Lemma 5. Suppose that $T \in \mathscr{L}(X)$ is Fredholm and has property $(\delta)$. Assume further that $S \in \mathscr{L}(Y)$ is such that there exist injective transformations $A \in \mathscr{L}(X, Y)$ and $B \in$ $\mathscr{L}(Y, X)$ with $A T=S A$ and $T B=B S$. Then there is a neighborhood $U$ of 0 and a positive integer $n$ such that $[T-\lambda]: X / \operatorname{ker}\left(T^{n}\right) \rightarrow X / \operatorname{ker}\left(T^{n}\right)$ is surjective for each $\lambda \in U$, and $S$ is Fredholm if and only if the quotient mapping $[S]$ on $Y / \operatorname{ker}\left(S^{n}\right)$ is Fredholm. Moreover, the quotient maps $X / \operatorname{ker}\left(T^{n}\right) \underset{[A]}{\stackrel{[B]}{\longrightarrow}} Y / \operatorname{ker}\left(S^{n}\right)$ are each injective and interwine $[T]$ and $[S]$.

Proof. Since $A$ and $B$ are injective and $\operatorname{ker}\left(T^{n}\right)$ is finite dimensional, $A$ and $B$ are bijections between $\operatorname{ker}\left(T^{n}\right)$ and $\operatorname{ker}\left(S^{n}\right)$. The last statement follows immediately.

Since $T^{*}$ has $(\beta)$, Lemma 4 gives $U \subset \rho_{e}(T)$ open about 0 and $n \in \mathbb{N}$ such that $\left.\left(T^{*}-\lambda\right)\right|_{\operatorname{ran}\left(T^{*}\right)^{n}}$ is injective for each $\lambda \in U$. We wish to show that each $[T-\lambda]$ has closed range. Thus suppose that $x \in X$ and $\left(x_{k}\right)_{k}$ is a sequence in $X$ such that $\left[(T-\lambda) x_{k}-x\right] \rightarrow 0$ as $k \rightarrow \infty$ in $X / \operatorname{ker}\left(T^{n}\right)$. Since $\operatorname{ran}(T-\lambda)$ is closed, we may, by the open mapping theorem, assume that the sequence $\left(x_{k}\right)_{k}$ is bounded in $X$. Choosing $\left(z_{k}\right)_{k} \subset \operatorname{ker}\left(T^{n}\right)$ such that $\left\|(T-\lambda) x_{k}-x+z_{k}\right\| \leq 2\left\|\left[(T-\lambda) x_{k}-x\right]\right\|$, it follows that $\left(z_{k}\right)_{k}$ is bounded in the 
finite dimensional space $\operatorname{ker}\left(T^{n}\right)$, and thus we may assume that there is a $z \in \operatorname{ker}\left(T^{n}\right) \operatorname{such}$ that $z_{k} \rightarrow z$ as $k \rightarrow \infty$. Therefore, $(T-\lambda) x_{k} \rightarrow(x-z)$, and $x-z \in \operatorname{ran}(T-\lambda)$.

Because $\operatorname{ker}\left(S^{n}\right)$ is finite dimensional, there is a finite dimensional subspace $E$ of $Y^{*}$

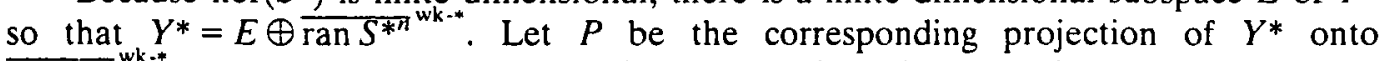
ran $S^{* \pi}{ }^{\mathrm{wk}-*}$. Then $S^{*}=S^{*} P+S^{*}(I-P)$, where $S(I-P)$ has finite rank. Since the collection of Fredholm operators on $Y^{*}$ is closed under compact perturbations, $S^{*}$ is Fredholm if and only if $S^{*} P$ is Fredholm. Since $\operatorname{ker}(P)$ is finite dimensional and

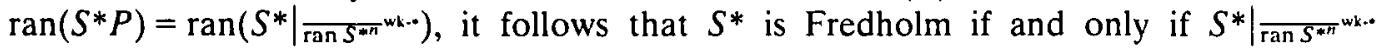
is Fredholm.

We now have all of the ingredients to prove the theorem.

THEOREM. Suppose that $T \in \mathscr{L}(X)$ and $S \in \mathscr{L}(Y)$ each have the decomposition property ( $\delta)$. If $A \in \mathscr{L}(X, Y)$ and $B \in \mathscr{L}(Y, X)$ are each injective, if $A T=S A$ and $T B=B S$, then $\sigma_{\mathrm{e}}(T)=\sigma_{\mathrm{e}}(S)$.

Proof. We need only show that $S$ is Fredholm provided that $T$ is Fredholm. Because the quotient of an operator with property $(\delta)$ itself has property $(\delta)$, and because of Lemma 5, we may assume that there is a neighborhood $V$ of 0 such that $T-\lambda$ is surjective and Fredholm for each $\lambda \in V$. We may also assume that $\operatorname{dim} \operatorname{ker}(T-\lambda)=$ ind $(T-\lambda)=\operatorname{dim} \operatorname{ker}(T)$ for all $\lambda \in V$. If $T$ is injective then $T$ is invertible and, by [12, Proposition 2.4], $S$ is also invertible. Otherwise, shrinking $V$ if necessary, there exist, by Lemma 1 , functions $f_{1}, \ldots, f_{n} \in \mathcal{O}\left(V, X^{*}\right)$ such that each $[f] \in \mathscr{F}_{T *}(V)$ can be written as $[f]=\sum \varphi_{j}\left[f_{j}\right]$ for some analytic functions $\varphi_{j}: V \rightarrow \mathbb{C}$. Lemma 3 now implies that $S^{*}$ is injective and that $\left\{\left[B^{*} f_{j}\right]\right\}_{j=1}^{n}$ generates $\mathscr{F}_{S^{*}}(V)$; i.e., for each $f \in \mathcal{O}\left(V_{n} Y^{*}\right)$ there exist analytic functions $\varphi_{j}: V \rightarrow \mathbb{C}$, and $g: V \rightarrow Y^{*}$ such that $f(\lambda)=\sum_{j=1}^{\lambda} \varphi_{j}(\lambda) B^{*} f_{j}(\lambda)+$ $\left(S^{*}-\lambda\right) g(\lambda)$, for each $\lambda \in V$. In particular,

$$
Y^{*}=\operatorname{span}\left\{B^{*} f_{j}(0)\right\}_{1}^{n} \oplus \operatorname{ran}\left(S^{*}\right)
$$

The theorem is established.

Clearly the previous result applies to quasisimilar operators with property $(\delta)$. Also, we immediately obtain the following results; see [11, Theorem 4.1.12].

Corollary. Suppose that $T \in \mathscr{L}(X)$ and $S \in \mathscr{L}(Y)$ each have Bishop's property $(\beta)$. If $A \in \mathscr{L}(X, Y)$ and $B \in \mathscr{L}(Y, X)$ each have dense range and satisfy $A T=S A, T B=B S$, then $\sigma_{e}(S)=\sigma_{e}(T)$.

ACKNOWLEDGEMENTS. An earlier version of this paper contained an error in the proof of Lemma 3. We are indebted to the referee for pointing it out and for other helpful suggestions. Joerg Eschmeier and Michael Neumann also very kindly offered comments which substantially improved this note.

\section{REFERENCES}

1. E. Albrecht and J. Eschmeier, Analytic functional models and local spectral theory, (submitted).

2. E. Bishop, A duality theorem for an arbitrary operator, Pacific J. Math., 9 (1959), 375-397.

3. W. S. Clary, Equality of spectra of quasi-similar hyponormal operators, Proc. Amer. Math. Soc. 53 (1975), 88-90. 
4. I. Colojoară and C. Foiaş, Theory of generalized spectral operators, (Gordon and Breach, New York, 1968).

5. J. Eschmeier, Analytische Dualität und Tensorprodukte in der mehrdimensionalen Spektraltheorie, Habilitationsschrift, Schriftenreihe des Math. Inst. Universität Münster, 2.42 (Münster, 1987).

6. J. K. Finch, The single valued extension property on a Banach space, Pacific J. Math. 58 (1975), 61-69.

7. A. Grothendieck, Sur certains espaces de fonctions holomorphes, I, II, J. Reine Angew. Math 192 (1953), 35-64, 77-95.

8. H. G. Heuser, Functional analysis, (John Wiley and Sons, New York, 1982).

9. H. Jarchow, Locally convex spaces (B. G. Teuber, Stuttgart, 1981).

10. G. Köthe, Topological vector spaces II (Springer-Verlag, 1979).

11. V. G. Miller, Restrictions and quotients of decomposable operators and spectral inclusions on Banach spaces, Thesis, Mississippi State University, 1993.

12. V. G. Miller and M. M. Neumann, Local spectral theory for multipliers and convolution operators, in Algebraic Methods in Operator Theory, Ed. R. Curto and P. Jørgensen (Birkhäuser, 1994), 25-36.

13. M. Putinar, Spectral theory and sheaf theory I, in Dilation Theory, Toeplitz Operators, and Other Topics (Birkhäuser, 1983), 283-298.

14. M. Putinar, Hyponormal operators are subscalar, J. Operator Theory 12 (1984), 385-395.

15. M. Putinar, Quasisimilarity of tuples with Bishop's property $(\beta)$, Int. Eq. and Oper. Theory, 15 (1992), 1048-1052.

16. M. A. Shubin, On holomorphic families of subspaces of Banach spaces, Int. Eq. and Oper. Theory, 2/3 (1979), 407-420.

17. F.-H. Vasilescu, Analytic functional calculus and spectral decompositions (Editura Academiei and D. Reidel Publishing Company, Burcureşti and Dordrecht, 1982).

Operator Theory 3 (1980), 57-69.

19. L. Yang, Equality of essential spectra of quasi-similar subnormal operators, Int. Eq. and Oper. Theory, 13 (1990), 433-441.

20. L. Yang Quasisimilarity of hyponormal and sub-decomposable operators, J. Functional Analysis, 112 (1993), 204-217.

\author{
Mississippi State UNiversity \\ DRAWER MA \\ Mississippi State \\ MS 39762 \\ U.S.A.
}

E-mail: miller@math.msstate.edu vivien@math.msstate.edu 